\title{
A Method for Optodynamic Characterization of Erbium Laser Ablation Using Piezoelectric Detection
}

\author{
Georgije Bosiger ${ }^{1, *}$ - Tadej Perhavec ${ }^{1}$ - Janez Diaci² \\ ${ }^{1}$ Fotona d.d. Ljubljana, Slovenia \\ 2 University of Ljubljana, Faculty of Mechanical Engineering, Slovenia
}

\begin{abstract}
The paper presents a new method for characterization of Erbium laser ablation processes widely employed in various medical applications. The method is based on detection of shock waves propagating in air above the irradiated surface by means of a wideband piezoelectric sensor and analysis of the acquired signal waveforms. This sensor set-up offers the possibility for integration into an Er:YAG laser hand-piece, which opens the way to on-line process monitoring.

A new model of the sensor is developed in order to take into account the relative position and orientation of the sensor and its mechanical and electrical properties. The model is verified by comparing the signal waveforms acquired at different sensor distances and orientations relative to the ablated spot with the theoretical waveforms calculated on the basis of numerical solutions of the Taylor-Sedov point explosion model and the developed sensor model. Excellent agreement is observed between the acquired and theoretical waveforms, which serves as a basis for a novel method that employs shock-wave energy released during the ablation process as a process characteristic that can be determined from the acquired signal waveforms. It is shown that shock-wave energy exhibits significantly less dependence on the position and orientation of the sensor than other waveform characteristics (time of fight, amplitude, etc.) that are currently used for the ablation process characterization.
\end{abstract}

Keywords: Erbium laser, laser ablation, shock wave, piezoelectric detection, Taylor-Sedov model

\section{INTRODUCTION}

The Er:YAG laser, with a wavelength of $2.94 \mu \mathrm{m}[1]$, is a well-established tool in medicine and surgery, particularly in dentistry [2] and dermatology [3]. Its infrared light is absorbed strongly in water and hydroxyapatite, providing effective laser ablation of soft and hard biological tissues [4]. Numerous new medical treatments (particularly in osteotomy) would also benefit from the advantages of Er:YAG laser tissue interaction over the conventional methods (e.g. noncontact intervention, smaller heat-affected zone, absence of mechanical vibration). One of the main technical and scientific challenges yet to be solved is the development of viable and reliable systems for the on-line monitoring of the key parameters, such as the cutting depth and the type of the removed tissue [5] to $[8]$.

Tissue ablation with the Er:YAG laser is driven by microexplosions. Absorbed laser energy is partially released in the form of nonlinear mechanical waves propagated in the ablated tissue and in the air above it. Following this, material ejection occurs. These so-called optodynamic phenomena have received considerable attention in the context of characterization of laser ablation. Various set-ups have been examined for this purpose: spatially resolved techniques, such as schlieren [9] and [10], shadowgraphy [11] to [14] or holography [15], as well as the temporally resolved ones: laser interferometer [16] to [18], laser beam deflection probe [19] and [20] and capacitive or piezoelectric acoustic sensors [7], [8] and [21]. While most of these techniques represent useful research tools within controlled laboratory experiments, only a few of them exhibit the potential to be used for on-line process monitoring in real medical applications. Here, additional technical factors come into prominence: e.g. compactness, affordability, insensitivity to environmental influences, speed of response, etc.

In our view, broadband piezoelectric acoustic sensors are the means to make the on-line process monitoring of Er:YAG laser ablation practicable. Fig. 1 illustrates the idea: a piezoelectric sensor is attached to the laser handpiece to detect shock waves in the air above the irradiated tissue. Considering typical conditions (geometry of the handpiece and sensor, focal length of the focusing optics, laser pulse energy, etc.) and previous research results [11] it is reasonable to assume that the shock waveform is nearly hemispherical as it impinges onto the sensor and its amplitude is decreased to the intermediate-to-weak shock level. Piezoelectric shock sensors that respond linearly to this kind of excitation are available.

Existing methods of shock wave characterization using acoustic sensors rely on empirically selected signal features, such as acoustic signal energy, signal amplitude and time of flight [7], [21] and [22]. Relationships of these signal features to other influencing factors (e.g. sensor characteristics, orientation or distance from the source) are not known, 
thus limiting the applicability of these methods to strictly controlled conditions.

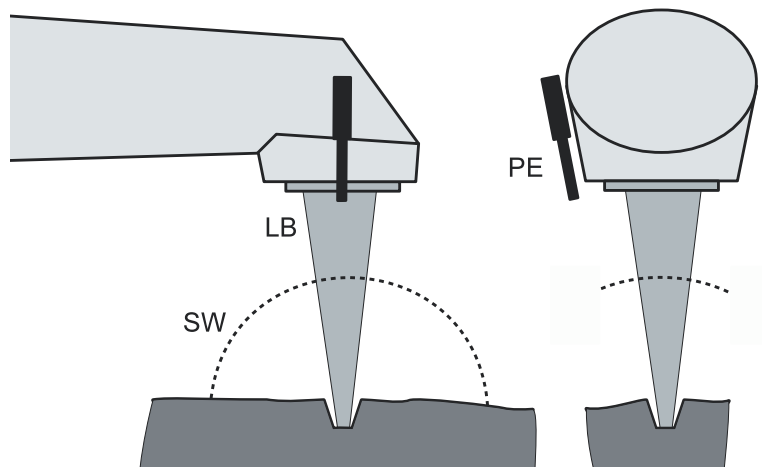

Fig. 1. Schematic representation of a set-up for optodynamic characterization of the laser ablation processes: shock wave (SW), piezoelectric sensor (PE), Er:YAG laser ablation beam (LB)

In this paper we propose another approach that opens the way to on-line process monitoring. We develop a new model of the sensor that takes into account the relative position and orientation of the sensor as well as its mechanical and electrical properties. We verify the model by comparing signals, detected at different sensor distances and orientations relative to the ablated spot, with the theoretical waveforms determined from a numerical solution of the point explosion model [23], taking into account the model of the piezoelectric sensor. Observing excellent agreement between the theoretical and experimental waveforms, we propose a novel method that employs shock-wave energy released during the ablation process as a process characteristic that is almost independent of the exact geometrical properties of the detection set-up.

\section{THEORY}

In this section, a new model of a piezoelectric shock sensor is presented. The finite size of the sensor and its mechanical and electrical characteristics are taken into account. Next, a numerical procedure is described that allows the determination of theoretical pressure waves at the sensor surface employing the TaylorSedov point explosion model.

\subsection{Piezoelectric Sensor Model}

The theoretical description is simplified on the assumption that the incident shock wave, during the propagation over the piezoelectric transducer surface, can be approximated as a linear spherical acoustic wave [24]. For a Taylor-Sedov explosion, this applies well for an intermediate or weak shock over a small distance of propagation. With this assumption, the pressure transient at a given point $r_{s}$ on the transducer can be expressed by the pressure in a centre point $r_{0}$ of the transducer, using the equation:

$$
\Delta p\left(t, r_{s}\right)=\frac{r_{s}}{r_{0}} \Delta p\left(t-\frac{r_{s}-r_{0}}{u_{0}}, r_{0}\right),
$$

where $u_{0}$ is the shock propagation speed which is determined using the point explosion model, and where it is expressed as a function of the distance from the source P and the released energy: $u_{0}=u_{0}\left(r_{0}, E\right)$.

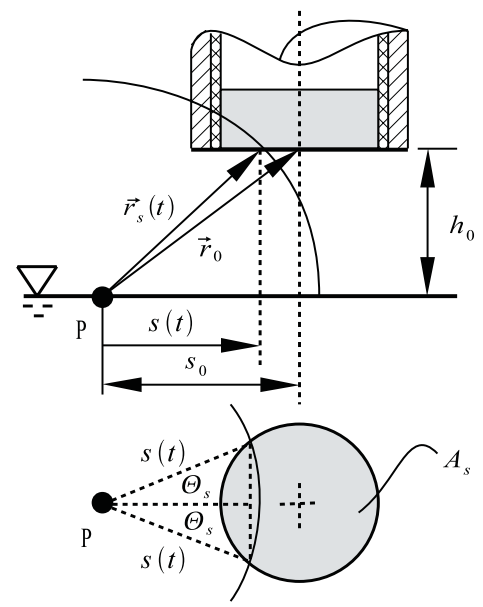

Fig. 2. Geometrical relations used in derivation of the sensor model - placement of the sensor relative to the ablation spot $(P)$ : side view (above), top view (below)

We also disregard all the effects that result due to a change of the acoustic impedance, assuming that these affect the amplitude and not the waveform. With this assumption we write:

$$
F_{a}\left(t ; \mathbf{r}_{\mathbf{0}}\right) \propto \int_{A_{s}} \Delta p\left(t, r_{s}\right) \mathrm{d} A_{s},
$$

where $F_{a}\left(t ; \mathbf{r}_{\mathbf{0}}\right)$ is the force that acts on the sensor in its axial direction, and $\Delta p\left(t, r_{s}\right)$ is the pressure transient at a point on surface $A_{s}$ of the sensor (see Fig. 2). Vector $\mathbf{r}_{\mathbf{0}}$ points to the sensor centre and can be expressed by the horizontal $s_{0}$ and vertical $h_{0}$ distance. Inserting Eq. (1) into (2) and substituting $\Delta p$ with a spherical impulse disturbance $\delta$, we get Rayleigh's integral [25]:

$$
h\left(t ; \mathbf{r}_{\mathbf{0}}\right)=r_{0} \cdot \int_{A_{s}} \frac{\delta\left(t-\frac{r_{s}-r_{0}}{u_{0}}, r_{0}\right)}{r_{s}} \mathrm{~d} A_{s} .
$$


The above equation represents the response of a sensor with a finite aperture to a spherical impulse disturbance. Assuming a cylindrical sensor with a flat aperture, it is possible to express $h\left(t ; \mathbf{r}_{0}\right)$ in terms of the angle $\Theta_{\mathrm{s}}\left(t ; \mathbf{r}_{\mathbf{0}}\right)$ determined by the intersection of the projected spherical wave onto the sensor aperture as shown on Fig. 2:

$$
h\left(t ; \mathbf{r}_{\mathbf{0}}\right)=2 u_{0} r_{0} \Theta_{s}\left(t ; \mathbf{r}_{\mathbf{0}}\right) .
$$

Using the superposition principle, we find that the force acting on a sensor of finite size is proportional to the convolution of a pressure transient $\Delta p\left(t, r_{0}\right)$ in the centre of the transducer and the signal waveform $\Theta_{\mathrm{s}}$ $\left(t ; \mathbf{r}_{\mathbf{0}}\right)$ representing the impulse response of the finite sensor aperture:

$$
F_{a}\left(t ; \mathbf{r}_{0}\right) \propto \Delta p\left(t, r_{0}\right) * \Theta_{s}\left(t ; \mathbf{r}_{0}\right) .
$$

In order to take into account the electrical and mechanical characteristics, we treat the sensor as a one-dimensional element, where deformation that generates electric current acts only in its axial direction. The basis for this assumption represents the design of the piezoelectric acoustic sensor, where the sensor housing and the insulation suppresses the radial excitations (see Fig. 2). Pure elastic deformations of the sensing element are assumed. With these assumptions, linear static and dynamic characteristics apply.

The Piezoelectric transducer is represented with a current source and capacitor $C_{S}$ in parallel [26]. Parallel resistance of the sensing element is usually large and can be neglected. Taking into account the capacitance of the cable $C_{c}$ and the resistive load $R_{L}$, we get a transfer function for a linear piezoelectric force sensing element, Eq. (6), where $\tau=R_{L}\left(C_{s}\right.$ $\left.+C_{c}\right)$ is the time constant, $d$ the piezoelectric charge constant and $V$ the measured voltage. $H(s)$ represents the dynamic characteristics of the elastic piezoelectric structure, where natural frequencies can be determined by measuring electrical impedance on an impedance analyzer [26].

By analyzing the transfer function in Eq. (6) we can conclude that the sensor's sensitivity in steady state is inversely proportional to the total capacitance. Due to this capacitance, the sensor behaves as a high pass RC filter with time constant $\tau$. The sensor output is in the frequency region between $1 /(2 \pi \tau)$ and the first natural frequency of the piezoelectric element is proportional to the force $F_{a}$.

$$
\frac{V(s)}{F_{a}(s)}=\frac{d}{c_{s}+c_{c}} \frac{\tau s}{1+\tau s} \frac{1}{H(s)} .
$$

\subsection{Shock-Wave Propagation Model}

We employ the Taylor-Sedov point explosion model to model propagation of the spherical shock wave [23]. The model assumes that a finite amount of energy $E$ is released instantly in an infinitesimal volume of a perfect gas. Propagation of the blast wave is described by a set of hyperbolic partial differential equations in the Euler form:

$$
\mathbf{U}_{t}+\mathbf{F}(\mathbf{U})_{r}=\mathbf{S}(\mathbf{U})
$$

where $\mathbf{U}$ is the vector of the conserved variables, $\mathbf{F}=\mathbf{F}(\mathbf{U})$ their fluxes and $\mathbf{S}(\mathbf{U})$ the geometric source term that results from the transformation of the Euler equations to the spherical coordinates. Subscripts denote partial derivatives with respect to the independent variables; time $t$ and radius $r$. Primitive variables are the mass density $\rho(r, t)$, fluid speed $v(r, t)$ and pressure $p(r, t)$. Propagation at shock wave-front $r=r_{s}(t)$ is governed by the Rankine-Hugoniot jump conditions.

Transition to dimensionless space $\xi=r / r_{c}$ and time $\tau=t / t_{c}=c_{0} \cdot t / r_{c}$ coordinates is performed [23] using the characteristic radius $r_{c}=\left(\alpha E / \kappa p_{0}\right)^{1 / 3}$ that depends on the released energy $E$, where $c_{0}$ is the sound speed, $\alpha=1.175$ and heat capacity ratio $\kappa=1.4$. Primitive variables are normalized with their respective values in the undisturbed gas. The solution thus becomes independent of the released energy and undisturbed gas state, therefore, numerical calculation can be performed once and scaling back into dimensional coordinates adapts it to the given conditions.

In the intermediate and weak shock range, only a numerical solution of the model is obtainable. We use an explicit discrete conservative numerical scheme [27]:

$$
\mathbf{U}_{i}^{n+1}=\mathbf{U}_{i}^{n}-\frac{\Delta t}{\Delta r}\left[\mathbf{F}_{i+1 / 2}-\mathbf{F}_{i-1 / 2}\right]+\Delta t \mathbf{S}_{i}
$$

where $\Delta t$ and $\Delta r$ denote time and spatial steps, respectively. The second order WAF finite volume explicit method is used for the calculation of the fluxes [27]:

$$
\mathbf{F}_{i+1 / 2}=\mathbf{F}\left(\overline{\mathbf{W}}_{i+1 / 2}\right) \text {, }
$$

where the mean average state equals:

$$
\begin{aligned}
\overline{\mathbf{W}}_{i+1 / 2} & =\frac{1}{2}\left(\mathbf{W}_{i}^{n}+\mathbf{W}_{i+1}^{n}\right)- \\
& -\frac{1}{2} \sum_{k=1}^{N} \operatorname{sign}\left(c_{k}\right) \varphi_{k}\left[\mathbf{W}_{i+1 / 2}^{(k+1)}+\mathbf{W}_{i+1 / 2}^{(k)}\right] .
\end{aligned}
$$


W denotes the vector of primitive variables and $c_{k}$ the Courant's number. Intercell states are computed with an approximate HLLC Riemann solver [27]. The Van Leer limiter $\phi$ is used to prevent numerical oscillations. Time integration is performed with the forward Euler method. An analytical solution of the strong shock theory serves as an initial condition for the numerical computation.

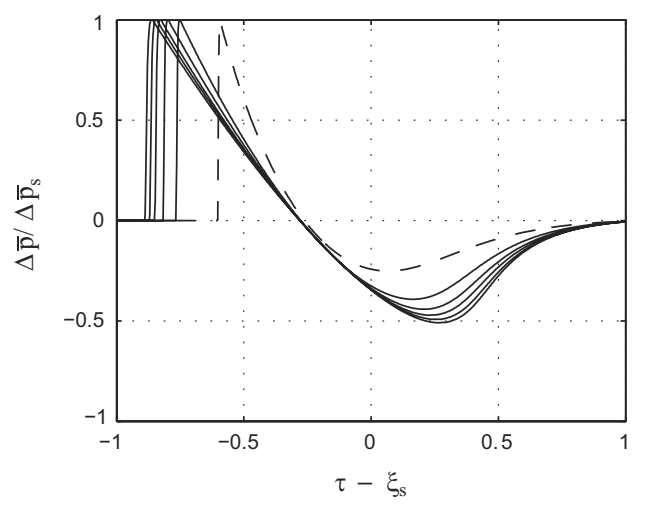

Fig. 3. Normalized theoretical waveforms retarded by the acoustic wave-front propagation time

Computation is performed over the dimensionless time interval $1 \leq \tau \leq 35$. The grid is equally spaced with 215 points for both independent variables. In the scope of this paper, only pressure is used. According to the model, the pressure transient in a given point is a function of two parameters - the distance from the source $r_{s}$ and the released energy $E$. Calculated pressure transients, normalized by the shock pressure amplitude, are shown in Fig. 3 for dimensionless distances $\xi_{s}: 2$ (dashed), 8, 14, 20, 26 and 32 on a time scale retarded by the time of flight of the acoustic wave-front to facilitate waveform comparison. Fig. 3 illustrates a common characteristic of spherical shock waves: the duration of the compression phase increases with distance $\xi_{s}$ due to supersonic shock wave-front propagation while the duration of the rarefaction phase remains constant.

\section{EXPERIMENT}

We use water as the tissue phantom for the purpose of stable and repeatable experimental validation. At the particular Erbium laser wavelength, water trapped or bonded to the tissue plays a key role in tissue ablation. Explosive expansion of laser-heated water generates strong shock waves that propagate in air above the ablated surface, followed by material ejection. The key difference between the ablation of water and the tissue is in the form of the surface on which the resulting shock waves are generated. Water and some soft tissues form a quasi-ideal half-space whereas hard tissues usually form complicated geometry that varies from pulse to pulse during the ablation and affects the spread of the shock wave. The results obtained by ablating the water surface are relevant for the laser surgery in which the shallow holes (craters) are prepared. For laser drilling of deep holes, an appropriate shock-wave propagation model has not yet been developed. In this case the accuracy of the presented method is questionable.

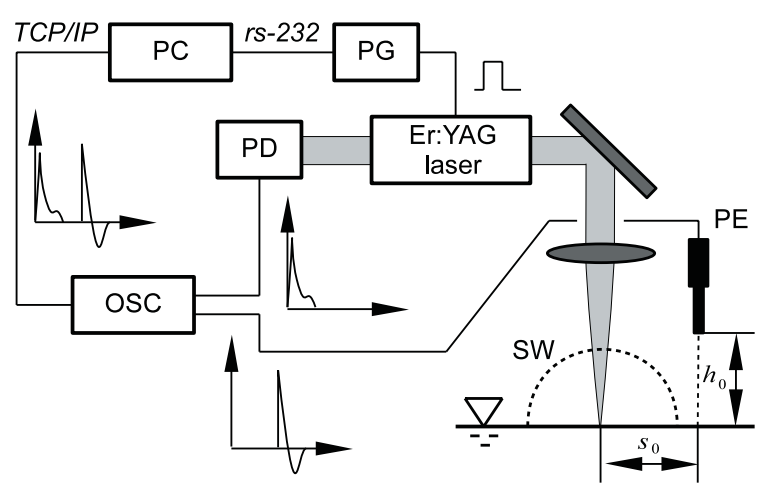

Fig. 4. Experimental set-up: shock wave (SW), piezoelectric sensor $(P E)$, photo-diode $(P D)$, oscilloscope (OSC, pulse generator $(P G)$, personal computer (PC)

The experimental system used to validate the above model is shown in Fig. 4. A free-running Er:YAG laser (Fidelis Plus III, Fotona) is used to irradiate the water surface as it forms a quasi-ideal half-space. The focal point of the laser exit optics is located on the water surface with a spot diameter of $0.9 \mathrm{~mm}$. A signal from a pulse generator triggers the laser system, also setting the pulse duration of the laser flash lamp $(45 \mu \mathrm{s})$. The supply voltage for laser pumping has been set to $650 \mathrm{~V}$. Resulting laser pulses are short $(\approx 2 \mu \mathrm{s})$, causing generation of a single shock wave rather than several that are typical for longer pulses [22]. The pulse energy was measured (Nova II, Ophir), where the mean value was $3.14 \mathrm{~mJ}$ with a std. deviation of $0.16 \mathrm{~mJ}$. A piezoelectric sensor (CA1135, Dynasen) with a PZT-5A crystal disc of $1 \mathrm{~mm}$ in diameter was translated in parallel and perpendicular to the water surface using two linear stages with micrometer screws.

Responses were measured at three different horizontal distances $(7.25,10.25$ and $13.25 \mathrm{~mm})$ and six vertical ones $(10,12,14,16,18$ and $20 \mathrm{~mm})$. Six repetitions on each configuration were conducted. The measured signals were sampled using a digital oscilloscope (Agilent DSO6034A) with $300 \mathrm{MHz}$ bandwidth and 12 bit digitization. Signal acquisition 
was triggered by a signal from an InAs photodiode (J12, Teledyne), mounted behind the back laser mirror. The signals were sent to a PC where they were saved. Room temperature and pressure were measured: $T_{0}=298 \mathrm{~K}, p_{0}=996 \mathrm{mbar}$, using standard meteorological equipment.

\section{RESULTS AND DISCUSSION}

We validate the described model by comparing the theoretical and measured waveforms. To enable this, the released energy is determined for every measured signal by measuring the duration of the compression phase of the measured signal.

Transforming the numerical solution of the point explosion to the dimensional coordinates and using the model of the sensor, we determine a function (Eq. 11) that describes the duration of the compression waveform phase $t_{p}$ as a function of characteristic radius $r_{c}$, given the influencing parameters: relative position of the sensor to ablation spot (determined by $s_{0}$ and $h_{0}$ ) and the sound speed $c_{0}$ which is estimated from the measured room temperature. The function is formulated as a spline with 41 data points (knots) along the characteristic radius $r_{c}$ for values between 0.8 and $2.8 \mathrm{~mm}$, whilst other parameters are held constant. At each data point a theoretical waveform (signal) is found first, from which time $t_{p}$ is then determined. The same procedure is repeated for other sensor positions.

$$
t_{p}=f\left(r_{c} ; s_{0}, h_{0}, c_{0}\right) .
$$

Using measured data for $t_{p}$ we numerically solve the above equation for $r_{c}$. Released energy for the spherical blast wave $E$ is then determined from the definition of the characteristic radius $r_{c}$. Duration $t_{p}$ is determined by normalizing the signal with its peak value and searching for the time interval where the normalized amplitude exceeds the threshold value of $0.1(10 \%)$. Signals are normalized because of simplifications of the theoretical model, where absolute signal values are unknown, Eq. (2), as the sensor is not calibrated.

The results are shown in Fig. 5. $E_{h}$ is the released energy for the half-space in which the shock wave forms a hemisphere $\left(E_{h}=E / 2\right)$. No systematic dependency of $E_{h}$ on sensor positions ( $s_{0}$, $h_{0}$, defined as shown in Figs. 2 and 4 ) is observed. It is to be noted that only sensor position $\left(s_{0}, h_{0}\right)$ varies in this experiment, while all other parameters (especially laser energy) remain the same. Pulse-topulse variations of $E_{h}$ within a sequence of repeated measurements, indicated by the error bars in Fig. 5, are mainly due to pulse-to-pulse variations of the laser pulse energy. We take the observation that $E_{h}$ does not exhibit systematic dependency on sensor position as evidence that the described model correctly describes the key features of the signals and the set-up. It is of interest to note the obtained $E_{h}$ value: its estimated mean value at $0.16 \mathrm{~mJ}$ (and std. dev. of $0.02 \mathrm{~mJ}$ ) implies that about $5 \%$ of the incident laser pulse is converted into the energy of the shock wave.

Using estimated released energies $E_{h}$, normalized theoretical signals are determined and compared to corresponding measured signal waveforms. Fig. 6 shows two limiting examples, characterized by high $\left(53^{\circ}\right)$ and low $\left(20^{\circ}\right)$ angles of incidence between the shock wave-front and the sensor aperture normal.

In both examples, good agreement of the positive (compressive) phase duration is observed between the theoretical and measured waveforms. This is
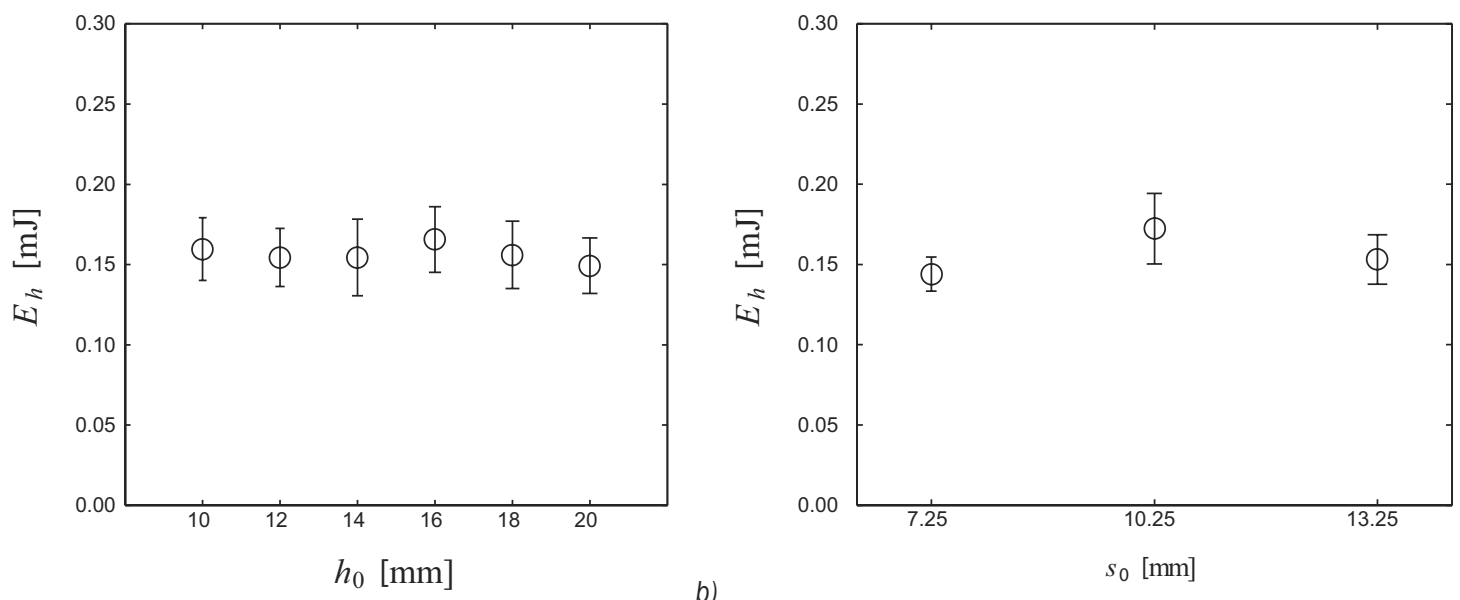

Fig. 5. Estimated energies for hemispherical explosion at a) senzor position $h_{0}$ and b) sensor position $s_{0}$ 
expected since this parameter was used to estimate released energy. In the negative (rarefaction) phase, the measured and theoretical signals differ. These differences are the consequence of various effects that are not taken into account by the presented model (e.g. arrival of water droplets and natural vibrations of the sensor structure, diffraction of the wave, etc.).

a)
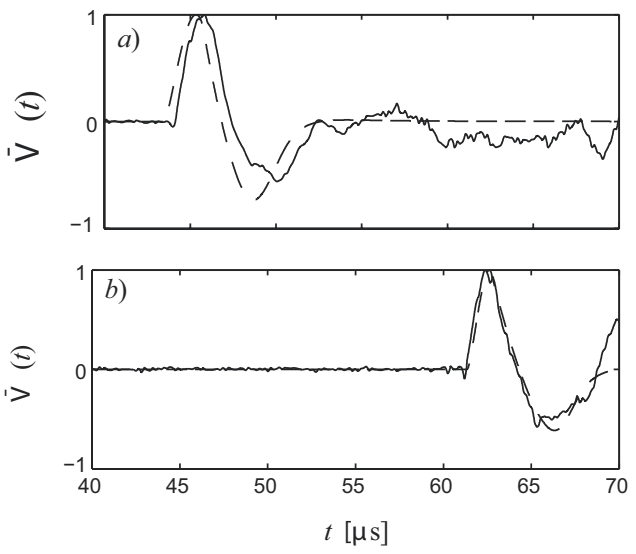

b)

Fig. 6. Comparison of measured and theoretical signals (dashed) for two different positions of the transducer relative to the ablation point, a) $\mathrm{s}_{0}=13.25 \mathrm{~mm}, h_{0}=10 \mathrm{~mm}$ and b) $s_{0}=7.25 \mathrm{~mm}, h_{0}=20 \mathrm{~mm}$

As a further step in model validation, we compare the theoretical and measured times of flight (TOF) of the shock wave-front. The maximum observed relative difference between a measured TOF and its theoretical counterpart is about $1 \%$. Expressing this in terms of distance using the theoretical shock speed, we get a maximum distance uncertainty of about $0.24 \mathrm{~mm}$. We attribute these deviations mainly to the uncertainties of the parameters in Eq. (11) and the measured durations $t_{p}$ that are used by the method for the estimation of the released energy $E_{h}$.

By comparing the theoretical shock waveforms that take into account the sensor model to the respective ones that do not, we find that the former ones systematically exhibit longer duration of the compressive phase. The relative difference between the two increases with the angle of incidence from $5 \%$ at the $20^{\circ}$ angle to $25 \%$ at the $53^{\circ}$ angle. This is a result of the prolonged transit time of the shock wave over the finite sensor surface in the case of a larger angle of incidence. This observation presents strong evidence that the finite dimensions of the sensor in given conditions need to be taken into account.

The described approach can be employed to calibrate the sensor. Peak pressures are found from the estimated energies $E_{h}$ using the point explosion model. Then, sensor sensitivity is calculated by dividing the peak voltage of a measured signal by the peak pressure of the corresponding theoretical signal. Analysis of the estimated sensor sensitivities shows that it varies significantly with sensor orientation $\left(s_{0}\right.$, $h_{0}$ ): the estimated pressure peaks in Fig. 6 are 17 and 12 mbar for the upper and lower trace, respectively, and the corresponding sensor sensitivities are 2.4 and $5.7 \mu \mathrm{V} / \mathrm{Pa}$, respectively. The observed variations of the estimated sensitivities at a fixed sensor position are less than $10 \%$.

\section{CONCLUSION}

We describe a method and a set-up that opens the possibilities for on-line process monitoring in Er:YAG laser ablation. We employ a piezoelectric shock sensor to detect shock waves generated in the air above the irradiated surface. Using a comprehensive sensor model and the Taylor-Sedov model of shock wave propagation, we demonstrate excellent agreement between the measured and theoretical waveforms. On this basis we propose that the released shock energy is a process characteristic that is essentially independent of the position and orientation of the sensor. We also demonstrate that this method allows peak shock wave pressure estimation and calibration of the sensor.

\section{ACKNOWLEDGEMENT}

The operational part of this research was financed by the European Union and the European Social Fund.

\section{REFERENCES}

[1] Bizjak, A., Nemeš, K., Možina, J. (2011). Rotatingmirror Q-switched Er:YAG laser for optodynamic studies. Strojniški vestnik - Journal of Mechanical Engineering, vol. 57, no. 1, p. 3-10, DOI:10.5545/svjme.2010.120.

[2] Bader, C., Krejci, I. (2006). Indications and limitations of Er:YAG laser applications in dentistry. American Journal of Dentistry, vol. 19, no. 3, p. 178-186.

[3] Hohenleutner, U., Hohenleutner, S., Bäumler, W., Landthaler, M. (1997). Fast and effective skin ablation with an Er:YAG laser: Determination of ablation rates and thermal damage zones. Lasers in Surgery and Medicine, vol. 20, no. 3, p. 242-247, DOI:10.1002/(SICI)1096-9101(1997)20:3<242::AIDLSM2>3.0.CO;2-Q.

[4] Niemz, M.H. (2007). Laser-Tissue Interactions: Fundamentals and Applications, 2nd ed. Springer, Berlin, Heidelberg.

[5] Burgner, J. (2010). Robot Assisted Laser Osteotomy. KIT Scientific Publishing, Karlsruhe. 
[6] Stübinger, S. (2010). Advances in bone surgery: the Er:YAG laser in oral surgery and implant dentistry. Clinical, Cosmetic and Investigational Dentistry, vol. 2, p. 47-62, DOI:10.2147/CCIDEN.S8352.

[7] Nahen, K., Vogel, A. (1999). Investigations on acoustic on-line monitoring of IR laser ablation of burned skin. Lasers in Surgery and Medicine, vol. 25, no. 1, p. 69-78, DOI:10.1002/(SICI)1096-9101(1999)25:1<69::AIDLSM9>3.0.CO;2-3.

[8] Rupprecht, S., Tangermann-Gerk, K., Wiltfang, J., Neukam, F.W., Schlegel A. (2004). Sensor-based laser ablation for tissue specific cutting: An experimental study. Lasers in Medical Science, vol. 19, no. 2, p. 8188, DOI:10.1007/s10103-004-0301-2.

[9] Apitz, I., Vogel, A. (2005). Material ejection in nanosecond Er:YAG laser ablation of water, liver, and skin. Applied Physics A, vol. 81, no. 2, p. 329-338, DOI:10.1007/s00339-005-3213-5.

[10] Nahen, K., Vogel, A. (2002) Plume dynamics and shielding by the ablation plume during Er:YAG laser ablation. Journal of Biomedical Optics, vol. 7, no. 2, p. 165-178, DOI:10.1117/1.1463047.

[11] Perhavec, T., Diaci, J. (2010). A novel double-exposure shadowgraph method for observation of optodynamic shock waves using fiber-optic illumination. Strojniški vestnik - Journal of Mechanical Engineering, vol. 56, no. 7 , p. 477-482.

[12] Gregorčič, P., Možina, J. (2011). High-speed two-frame shadowgraphy for velocity measurements of laserinduced plasma and shock-wave evolution. Optics Letters, vol. 36, no. 15, p. 2782-2783, DOI:10.1364/ OL.36.002782.

[13] Gregorčič, P., Diaci, J., Možina, J. (2012). Twodimensional measurements of laser-induced breakdown in air by high-speed two-frame shadowgraphy. Applied Physics A, p. 1-7.4.

[14] Gregorcic, P., Jezersek, M., Mozina, J. (2012). Optodynamic energy-conversion efficiency during an Er:YAG-laser-pulse delivery into a liquid through different fiber-tip geometries. Journal of Biomedical Optics vol. 17, no. 7, p. 075006-1, DOI:10.1117/1. JBO 17.7.075006.

[15] Amer, E., Gren, P., Sjodahl, M. (2008). Shock wave generation in laser ablation studied using pulsed digital holographic interferometry. Journal of Physics D: Applied Physics, vol. 41, p. 215502, DOI:10.1088/0022-3727/41/21/215502.
[16] Požar, T., Možina, J. (2009). Homodyne quadrature laser interferometer applied for the studies of optodynamic wave propagation in a rod. Strojniški vestnik - Journal of Mechanical Engineering, vol. 55, no. 10, p. 575-580.

[17] Požar, T., Gregorčič, P., Možina, J. (2009). Optical measurements of the laser-induced ultrasonic waves on moving objects. Optics Express, vol. 17, no. 25, p. 22906-22911, DOI:10.1364/OE.17.022906.

[18] Požar, T., Gregorčič, P., Možina, J. (2011). A precise and wide-dynamic-range displacement-measuring homodyne quadrature laser interferometer. Applied Physics B: Lasers and Optics, vol. 105, no. 3, p. 575582, DOI:10.1007/s00340-011-4512-5.

[19] Diaci, J., Možina, J. (1995). Multiple-pass laser beam deflection probe for detection of acoustic and weak shock waves in fluids. Review of Scientific Instruments, vol. 66, p. 4644-4648, DOI:10.1063/1.1145301.

[20] Diaci, J., Mozina, J. (1994). A study of energy conversion during Nd:YAG laser ablation of metal surfaces in air by means of a laser beam deflection probe. Le Journal de Physique, vol. 4, p. 737-740.

[21] Lukac, M. Grad, L., Mozina, J., Sustercic, D., Funduk, N., Skaleric, U. (1994). Optoacoustic effects during Er:YAG laser ablation in hard dental tissue, Proceedings SPIE 2327, Medical Applications of Lasers II, p. 93100.

[22] Grad, L., Možina, J. (1996). Optodynamic studies of Er:YAG laser induced microexplosions in dentin. Applied Surface Science, vol. 96-98, p. 591-595, DOI:10.1016/0169-4332(95)00559-5.

[23] Sedov, L.I. (1993). Similarity and Dimensional Methods in Mechanics, 10th Ed.. CRC Press, Boca Raton.

[24] Kinsler, L.E., Frey, A.R. (1962). Fundamentals of Acoustics. Wiley, New York.

[25] Jensen, J.A. (1999). A new calculation procedure for spatial impulse responses in ultrasound. Journal of the Acoustical Society of America, vol. 105, no. 6, p. 3266-3274, DOI:10.1121/1.424654.

[26] Bentley, J.P. (2005). Principles of Measurement Systems. Pearson Education, Essex.

[27] Toro, E.F. (2009). Riemann Solvers and Numerical Methods for Fluid Dynamics: A Practical Introduction. Springer, Dordrecht, DOI:10.1007/b79761. 